\title{
The Formation of Topological Defects in Phase Transitions
}

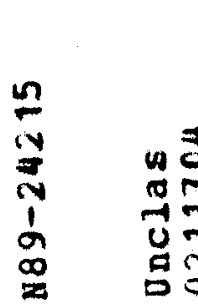

$m$

\author{
Hardy M. Hodges \\ Department of Physics \\ The University of Chicago \\ Chicago, IL 60637 \\ and
}

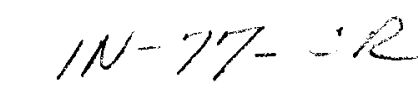

NAGW-1S:O

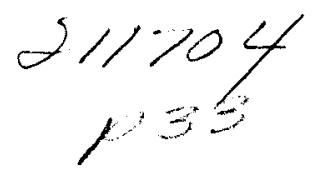

NASA/Fermilab Astrophysics Center

Fermilab, P.O. BOX 500, Batavia, IL 60510

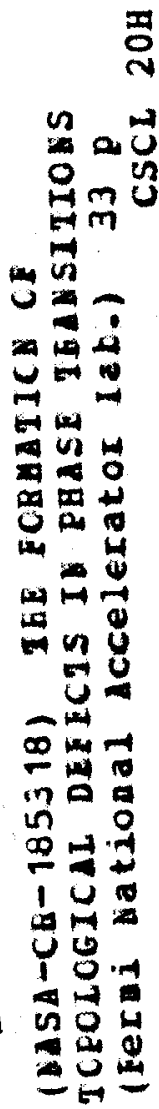

\begin{abstract}
We argue, and find through numerical work, that the results of non-dynamical Monte Carlo computer simulations cannot be applied to describe the formation of topological defects when the correlation length at the Ginzburg temperature is significantly smaller than the horizon size, the case which was originally considered. To test the current hypothesis that 'infinite' strings at formation are essentially described by Brownian walks of size the correlation length at the Ginzburg temperature, we examine equilibrated fields at the Ginzburg temperature. We find that no 'infinite' structure exists in equilibrium for reasonable definitions of the Ginzburg temperature, and that horizons must be included in a proper treatment. A phase transition, from small-scale to large-scale string or domain wall structure, is found to occur very close to the Ginzburg temperature, in agreement with recent work. We also investigate the formation process of domain walls and global strings through the breaking of initially ordered states. To mimic conditions in the early Universe, cooling times are chosen so that horizons exist in our sample volume when topological structure formation occurs. The classical fields are evolved in real-time by the numerical solution of Langevin equations of motion on a three dimensional spatial lattice. Our results indicate that it is possible for most of the string energy to be in small loops, rather than in long strings, at formation.
\end{abstract}

Present address: Santa Cruz Institute for Particle Physics, University of California, Santa Cruz, CA 95064 . 


\section{Introduction}

About a decade ago, some important implications of cosmological phase transitions were realized. The possibility that a discrete symmetry in the early Universe became broken, resulting in domain structure, was discussed by Zel'dovich et al [1]. Shortly thereafter, Kibble [2] classified topologically stable defects, and discussed their subsequent evolution in the Universe. To review, the essential idea behind defect formation is that below a critical temperature degenerate vacuum states are realized. The most direct argument for the formation of such defects is then based on causality - which requires that different horizon volumes end up in uncorrelated vacuum states. Depending upon the symmetry that is being broken, regions of the old, symmetric vacuum state can be trapped in point defects (monopoles), line defects (strings), and planar defects (walls) as a consequence of neighboring horizons being in different vacuum states. More complicated objects, such as walls bounded by strings, and monopoles connected by strings can also form. In the typical field theories describing these objects, their continued existence in the Universe, after the phase transition, is purely due to non-thermal effects. That is, the ground state of the theory at zero temperature is where all of space is in just one vacuum state, and there are no topological defects- an unattainable state, however, as long as there are horizons.

Of all the defects, strings have received special attention as they can produce the density fluctuations required for galaxy formation [3], provided the energy per length $\mu$ is $G \mu \approx 10^{-6}$ (a typical GUT scale). The success of the scenario hinges upon whether or not a scaling solution exists, i.e., whether or not long strings can efficiently lose energy through loop production to avoid a string-dominated universe. Much work has gone into numerical simulations of Nambu strings in an expanding Universe [4] to determine if such a solution exists-the evidence suggests there is a scaling solution. To better understand the properties of strings, the distribution of strings at formation has been modeled by using a non-dynamical Monte Carlo simulation-which is also used as initial conditions in the simulations. In addition, equilibrium properties of strings has been studied via analytic treatments of string statistical mechanics and by computer simulation. However, all previous models of formation lack a notion of dynamics, an essential ingredient of the formation process.

The formation of cosmic strings has been extensively studied using non-dynamical Monte Carlo simulations $[5,6,7]$. In these simulations, space is discretized on a latice and the phase $\chi$ of a complex Higgs field $\Phi\left(=f e^{i \chi}\right.$, where $f$ and $\chi$ are real $)$ is randomly

assigned values $[0,2 \pi]$ to the lattice sites (or, alternatively, to the cells) which represent 
regions of constant phase. A frequent approximation, which is really not necessary, is to discretize the infinite number of vacuum states into a few possibilities (e.g. $\chi=0$, $2 \pi / 3,4 \pi / 3)$. Changes in phase $\Delta \chi$ between neighboring sites are taken minimally, as gradients in the Higgs field cost kinetic energy. Upon traversing any closed path, the net phase change $\Delta \chi$ is $2 \pi N$, where $N$ is an integer (the winding number). Strings, which must either be closed or have ends on the spatial boundary, are then easily found by checking each lattice face for a non-zero winding number. On a cubic lattice, following the above procedure for the identification of a string, $|N| \leq 1$. If more than one string enters (and exits) a cell, the incoming and outgoing strings are randomly connected. The results of such a simulation indicate that $\simeq 80 \%$ of the string length is in long strings ( $\gtrsim$ simulation dimensions), with the remaining $\simeq 20 \%$ in 'small' loops with a scale-invariant distribution. For reference, and further comparisons, we plot the distribution of strings obtained using this technique, for one run on a $35^{3}$ lattice, in Fig. 1.

For the above model to be self-consistent, the lattice sites must be separated by a length $\Delta$ over which the phase $\chi$ is uncorrelated. The horizon length provides an upper bound to $\Delta$. However, if one takes $\Delta$ to be the horizon length, then the largescale structure of the string distribution is obtained (assuming $\chi$ does not fluctuate too much on scales $<<\Delta$ ), but all information on sub-horizon structure is lost. It has been suggested [5] that the full string distribution, at formation, could be obtained by identifying the lattice spacing $\Delta$ with the correlation length $\xi_{G}$ determined from spatial variations in $\Phi$ at the Ginzburg temperature $T_{G}$. The strings then essentially have the shape of Brownian trajectories of persistence length $\xi_{G}$ at formation [5]. [For a good review of the heuristic picture of string formation, see Ref. [8].] However, we point out that it is not clear that their interpretation of the lattice spacing as $\xi_{G}$ is consistent with their model of randomly assigning phases. Troublesome points are: (1) $\xi_{G}$ does not soley describe correlations in $\chi$; it also reflects spatial fluctuations in the magnitude of $\Phi$, i.e., $f(2)$ there are two physical length scales that arise in this problem, the correlation length $\xi$ and the horizon size $d$, yet their picture only involves $\xi(3)$ since horizons do not explicitly enter in their model, we might extrapolate their results to describe equilibrium configurations of strings; however, if the fields in a very large volume were completely thermalized, the symmetry should be broken at the Ginzburg temperature-in contrast to what is obtained by randomly assigning phases with equal probability. The formation picture given by Vachaspati and Vilenkin is very appropriate for the case $\xi_{G} \simeq d$, yet this is not the case they had in mind $\left(\xi_{G}<<d\right)$, nor does it represent an interesting dynamical range in the parameters $\xi_{G}, d$. It is certainly plausible that the horizon length scale could have little effect on the initial string distribution for $\xi_{G}<<d$, e.g. if thermal fluctuations 
in the Higgs phase are sufficient at the Ginzburg temperature to produce large-scale structure, but this must be checked (we shall find that the horizon plays an extremely important role).

To elucidate the details of topological structure formation, we numerically solve finite-temperature equations of motion, describing both domain walls and global strings, with additional noise and damping terms which represent coupling to a heat bath. The equilibrium defect distribution is examined at various temperatures and, in particular, at the Ginzburg temperature $T_{G}$. In addition, we model dynamics by preparing ordered states and then cooling to $T_{G}$, where the structure is examined, on time scales that allow horizons to be present in the sample volume. We, therefore, simulate, and numerically verify, the 'Kibble mechanism' for the production of topological defects. A number of questions are also addressed. For example, what qualifications, if any, should be placed on the presently held view that $80 \%$ of the length in strings at formation is in long strings? Is $\xi_{G}$ the only relevant scale in determining the string distribution, as has been suggested? If the horizon does in fact play a role, what does the string distribution look like at formation if the correlation length at the Ginzburg temperature is much smaller than the horizon?

The paper is organized as follows: in Sec II we provide the details of our approach and examine the properties of domain walls at formation; in Sec III we examine the properties of global strings at formation; in Sec IV we summarize our work and make some concluding remarks. Both Sec II and Sec III are subdivided into equilibrium (no horizons) and non-equilibrium (horizons) sections. The equilibrium sections test the standard hypothesis that infinite strings or walls can exist at the Ginzburg temperature even when there are no horizons. In the non-equilibrium sections, horizons are introduced to investigate their role in the formation process, as we shall determine in the equilibrium sections that the standard hypothesis needs modification.

\section{Formation of Domain Walls}

\section{A. Equilibrated Fields}

We first consider the simplest topological defect, the domain wall, which is characterized by one real scalar field $\sigma$ and a zero-temperature bistable potential $V(\sigma)$, which we take to be $V(\sigma)=\lambda\left(\sigma^{2}-\eta^{2}\right)^{2} / 4$ !. At finite temperatures, the effective potential $V_{T}(\phi)$, where $\phi$ is the classical part of $\sigma$, becomes relevant. The exact nature of the temperature-dependence in the model is not very important here, and we sim- 
ply choose a Ginzburg-Landau type potential with $V_{T}(\phi)=-m^{2} \tau \phi^{2} / 2+\lambda \phi^{4} / 4$ ! + constant, where $\tau \equiv 1-\left(T / T_{c}\right)^{2}$ and $T_{c} \simeq 2 \eta$ ( $m$ and $\lambda$ are taken to be constants). We could just as easily take a phenomenological viewpoint and consider the form originally studied by Landau $\left(\tau=1-T / T_{c}\right)$, but choose the other form as it represents the leading temperature dependence in the high temperature expansion of the full one loop finite-temperature potential calculated from $V(\sigma)$, from which one finds $T_{c} \simeq 2 \eta[9]$. [To be consistent with this approximation, that the temperature be significantly larger than the mass, we stay reasonably close to the critical temperature in our simulations.] At temperatures above the critical temperature $T_{c}$, an ordered state $\langle\phi\rangle=0$ exists, but as the temperature is dropped below $T_{c}$ the $\phi \leftrightarrow-\phi$ discrete symmetry will be broken, and at $T=0$ either $\langle\phi\rangle=-\eta$ or $\langle\phi\rangle=+\eta$. In the early Universe, however, thermal contact is limited to a horizon volume. This means that the $\phi \leftrightarrow-\phi$ symmetry will be restored on scales larger than the horizon. The regions that separate the two distinct vacua are domain walls, and their large-scale existence is guaranteed as long as complete thermalization cannot take place, e.g. there are horizons. The classical field theory solution of a domain wall in the $\mathrm{x}-\mathrm{y}$ plane at zero temperature is $\phi=\eta \tanh (z \mathrm{~m} / \sqrt{2})$, which yields a wall width $w \approx \sqrt{2} m^{-1}[m=\sqrt{\lambda} \eta / \sqrt{2}$, the scalar mass $]$. Operationally, the classical solution is a useless definition of a domain wall. We shall simply define a wall to be a surface with $\phi=0$, at any temperature.

To study the formation of domain walls, we solve the (flat space) classical equation of motion with two additional semi-phenomenological terms $\Gamma \dot{\phi}$ and $\zeta$ :

$$
\ddot{\phi}+\Gamma \dot{\phi}-\nabla^{2} \phi+\partial V_{T}(\phi) / \partial \phi=\zeta
$$

where dots denote time derivatives, and the field $\zeta$ is a noise term, controlled by an amplitude $A$, which represents coupling to a heat bath and has the following properties:

$$
\begin{aligned}
& <\zeta(x, t)>=0 \\
& <\zeta(x, t) \zeta\left(x^{\prime}, t^{\prime}\right)>=2 \Gamma A \delta^{3}\left(x^{\prime}-x\right) \delta\left(t^{\prime}-t\right)
\end{aligned}
$$

$\Gamma \dot{\phi}$ is a dissipative term in the equation of motion, which must be present if finitetemperature equilibrium configurations are to be established. In an expanding Universe such a damping term arises naturally; however, without loss of the physics at hand, we do not consider expansion and we choose $\Gamma$ to be a constant. Expansion would have several qualitative effects: (1) damping, given by $\Gamma=1 / 2 t$ in a radiation 
dominated Universe, (2) horizons of size $2 t$, and (3) redshifting of the $\nabla^{2} \phi$ term in Eqn. (2.1) by a factor $\propto t^{-1}$. Since we are presently testing the standard argument that infinite structure can result from the equilibrium distribution at the Ginzburg temperature, ignoring horizons, we certainly do not care about the expansion effects (2) and (3). The only crucial property in this investigation is damping (so equilibrium configurations can be achieved), which need not even be supplied by the expansion of the Universe. Damping will also occur due to the frictional effects associated with the coupling of $\phi$ to other fields. On dimensional grounds, we simply take $\Gamma \sim m_{\text {eff }}$, where the effective mass $m_{\text {eff }}=m \sqrt{\tau}$. The choice of $\Gamma$, for a fixed temperature, will only affect the probability distribution of the $\phi$ field when we consider non-equilibrium field configurations (see below). In any case, the damping rate chosen here will not significantly differ from the expansion rate for parameters of interest.

Eqn (2.1) is analytically insoluble, even without the additional terms, so we must numerically solve a discretized version of the equation of motion. We obtain the equations of motion from the lattice Hamiltonian $H$, which we take to be:

$$
\begin{aligned}
H= & \sum_{i, j, k=1}^{N}\left[\frac{\pi_{i, j, k}^{2}}{2 \Delta^{3}}+\frac{\Delta}{2}\left[\left(\phi_{i+1, j, k}-\phi_{i, j, k}\right)^{2}+\left(\phi_{i, j+1, k}-\phi_{i, j, k}\right)^{2}+\left(\phi_{i, j, k+1}-\phi_{i, j, k}\right)^{2}\right]\right. \\
& \left.+\Delta^{3} V\left(\phi_{i, j, k}\right)\right]+\frac{\pi_{N+1, N+1, N+1}^{2}}{2 \Delta^{3}}+\Delta^{3} V\left(\phi_{N+1, N+1, N+1}\right)
\end{aligned}
$$

where $\Delta$ is the cubic lattice spacing, and the conjugate momenta $\pi_{i, j, k}=\Delta^{3} \dot{\phi}_{i, j, k}$. Lattice sites are located at $i, j, k=1, \ldots, N+1$ in a total sample volume $V=L^{3}=$ $N^{3} \Delta^{3}$. Our phenomenological equations of motion for the lattice fields $\phi_{i, j, k}$ are then given by:

$$
\ddot{\phi}_{i, j, k}=-\Delta^{-3} \partial H / \partial \phi_{i, j, k}-\Gamma \dot{\phi}_{i, j, k}+\zeta_{i, j, k}
$$

and we now have $\left\langle\zeta_{i, j, k}\right\rangle=0$ and $\left\langle\zeta_{i, j, k}(t) \zeta_{l, m, n}\left(t^{\prime}\right)\right\rangle=2 \Gamma A \delta_{i l} \delta_{j m} \delta_{k n} \delta\left(t-t^{\prime}\right) / \Delta^{3}$. If the noise terms $\zeta_{i, j, k}$ are drawn from a Gaussian distribution, the Fokker-Planck equation [10], which describes the probability $p$ of measuring a given configuration $\left\{\phi_{i, j, k}\right\}$, has the stationary solution:

$$
p \propto e^{-F / A}
$$

where $F$ is the discrete version of the usual Ginzburg-Landau free energy functional ( $\left.=\int d^{3} x\left[(\nabla \phi)^{2} / 2+V_{T}(\phi)\right]\right)$. Therefore, we henceforth identify the amplitude $A$ with the temperature $T$. The damping parameter $\Gamma$ does not explicitly appear in the $\phi$ distribution at long times, which should not be surprising as the equilibrium distribution should be independent of the dissipation time scale. The most probable 
field configuration $\left\{\phi_{i, j, k}\right\}$, obtained from $\partial F / \partial \phi_{i, j, k}=0$, is when all the lattice fields $\phi_{i, j, k}=\eta \sqrt{\tau}$ for $T<T_{c}$, and $\phi_{i, j, k}=0$ for $T>T_{c}$. A second-order phase transition is therefore expected near $T_{c}$.

Before proceeding further, we find it very convenient to define, and use exclusively, the following dimensionless fields/parameters: $P \equiv \phi / \eta, \tilde{\Gamma} \equiv \Gamma / m, \tilde{t} \equiv t m, \tilde{\Delta} \equiv \Delta m$, $\tilde{T} \equiv m T / \eta^{2}$, and $R \equiv \partial P / \partial \tilde{t}$. [In addition, we use units where $\hbar=c=k_{B}=1$ throughout.] We solve the two first-order equations associated with Eqn. (2.5) via Eulerian integration. The difference equations, in our dimensionless units, are:

$$
\begin{aligned}
& P_{i, j, k}(\tilde{t}+\epsilon)=P_{i, j, k}(\tilde{t})+\epsilon R_{i, j, k}(\tilde{t}) \\
& R_{i, j, k}(\tilde{t}+\epsilon)=R_{i, j, k}(\tilde{t})-\epsilon\left(G_{i, j, k}(\tilde{t})+\tilde{\Gamma} R_{i, j, k}(\tilde{t})+P_{i, j, k}(\tilde{t})\left[P_{i, j, k}^{2}(\tilde{t})-\tau\right]\right)+\alpha_{i, j, k}(\tilde{t})(28
\end{aligned}
$$

where $G_{i, j, k} \equiv\left[6 P_{i, j, k}-P_{i+1, j, k}-P_{i-1, j, k}-P_{i, j+1, k}-P_{i, j-1, k}-P_{i, j, k+1}-P_{i, j, k-1}\right] / \tilde{\Delta}^{2}, \epsilon$ is the time step, and $\left\langle\alpha_{i, j, k}\right\rangle=0,\left\langle\alpha_{i, j, k}(\tilde{t}) \alpha_{l, m, n}(\tilde{t}+h \epsilon)\right\rangle=2 \epsilon \tilde{T} \tilde{\Gamma} \delta_{h 0} \delta_{i l} \delta_{j m} \delta_{k n} / \tilde{\Delta}^{3}$. We further define a dimensionless amplitude $w_{i, j, k}$ through the relation $\alpha_{i, j, k}(\tilde{t})=$ $w_{i, j, k}(\tilde{t}) \sqrt{24 \tilde{T} \tilde{\Gamma} \epsilon / \tilde{\Delta}^{3}}$. To satisfy the discretized versions of Eqns. (2.2) and (2.3), we assign $w_{i, j, k}$ at each lattice site, every time step, a randomly chosen listing $Q$ from a library of 50000 Gaussian distributed numbers with $\langle Q\rangle=0,\left\langle Q^{2}\right\rangle=1 / 12$.

A similar Langevin approach can be found in Ref. [11], where the two-dimensional $X Y$ model, a model for both liquid-helium films and smectic-C liquid crystals, is studied. In particle physics, Langevin techniques have been used as an alternative to Monte Carlo simulations of lattice field theories [12]. In this case, the Langevin time has no physical meaning-it is simply an artificial extra dimension used to evolve field configurations to a state of thermal equilibrium. These techniques have also recently been used in cosmology to study the effects of fluctuations on the dynamics of the inflaton [13]. In these studies, as is the case here, we can view the Langevin time as 'real' time.

We begin by mapping out the phase structure of the field $\phi$ on the lattice. Since the correlation length $\xi \propto 1 / \sqrt{|\tau|}$ (see, e.g. [14]), near the phase transition, we choose a temperature-dependent lattice spacing $\tilde{\Delta}=1 / \sqrt{|\tau|}$. Nowhere shall we be concerned with variations in $\phi$ on scales smaller than the coherence length $\xi$, so the cutoff used here is both computationally sound and physically reasonable. To examine phase structure, we define an order parameter $\psi$ :

$$
\psi=|\langle P\rangle|
$$


which describes a completely broken state if $\psi=1$, and the state of full $(\phi \leftrightarrow-\phi)$ symmetry if $\psi=0$. Although $\phi$ is indeed an order parameter, it is more convienent to use $\psi$ as it provides a vacuum-independent description of the transition (e.g., depending on the initial conditions of a run, $\langle\Phi\rangle$ could be either positive or negative).

Thermal averages of local variables (e.g. $P, R$ ) are obtained from time averages, and translational invariance:

$$
\begin{aligned}
& <f>=\frac{1}{\tilde{t}_{\max }} \int_{0}^{\tilde{t}_{\max }} f d \tilde{t} \\
& f=\frac{1}{N^{3}} \sum_{i, j, k=1}^{N+1} f_{i, j, k}
\end{aligned}
$$

Measurements should be taken on time scales $\tilde{t}_{\max }>>\tilde{\Gamma}^{-1}$. In addition, because information travels at a finite speed $c=1$ in this model, we further require $\tilde{t}_{\max }>>$ $N \tilde{\Delta}$ so that all regions in the sample volume will have communicated with each other for a sufficiently long time (this condition is not essential, however, if the initial conditions are judicously chosen). We have taken $\epsilon=\tilde{\Delta} / 6$ and $\tilde{\Gamma}=3 \sqrt{\tau}$, throughout. We have also chosen the case $\tilde{T}_{c}=0.1$, which is equivalent to choosing $\lambda=1.5 \times 10^{-2}$. A $20 \times 20 \times 20$ lattice, with periodic boundary conditions, was used to explore $\psi$ and $\left.<\phi^{2}\right\rangle$ as a function of temperature. To quickly establish equilibrium, we initially chose $P_{i, j, k}$ equal to the expected mean field value, and took $R_{i, j, k}=0$. [The initial conditions are irrelevant in determining the long-time statistical properties, but other initial conditions were used as a check.] The results are shown in Figs. 2, 3. Typically, equilibrium configurations were reached after $\sim 100$ time steps, but several thousand time steps were taken in a given run. Uncertainties, and averages, were obtained from statistically independent "measurements" obtained from binning the data into bins of width the correlation time. From Fig. 2 we see that $\psi$ appears to be a continuous order parameter, and as expected indicates a second-order phase transition with $\tilde{T}_{c} \simeq 0.1$. The mean field theory result $\bar{P}=\sqrt{\tau}\left(T<T_{c}\right)$ is also plotted in Fig. 2, in agreement with our numerical results ouside of the critical region. In Fig. 3 we have plotted $<(P-<P>)^{2}>/<P>^{2}$ as a function of the temperature.

The equilibrium properties of domain walls was also examined as a function of temperature. Wall segments were located by checking if $\phi_{i, j, k}$ changed sign between neighboring lattice sites. All wall segments must either connect to form closed surfaces, or open surfaces terminating on the lattice boundary [we refer to a given surface as a domain wall]. The total domain wall area in the sample volume was calculated 
as a function of temperature, and is shown in Fig. 4. Averages, and error bars, of the total wall area were calculated from three statistically independent field configurations. The domain wall area rapidly increases near the transition temperature. The transition from the broken phase to the symmetric $\langle\phi\rangle=0$ phase, from a topological viewpoint, is then due to the rapid increase of domain wall area near the transition temperature-eventually they saturate space and $\langle\phi\rangle=0$ is achieved. The number of domain walls, of a given size, as a function of temperature was also calculated. Below $\tilde{T}=0.07$ we found no walls in our runs. For $0.09<\tilde{T}<0.094$ we found 0-13 walls, in a given run, of the minimum allowed size (6 sides). As the temperature was increased beyond this (to $\tilde{T}=0.0995$ ), the very small walls became more numerous, but at the same time the wall area in small walls compared to that in large walls became increasingly smaller.

We have ceased describing the wall distribution beyond $\tilde{T}=0.0995$ as it is sensible to describe structure, e.g. a domain wall, only when the fluctuations in the field $\phi$ are not too large. We, therefore, concentrate on examining structure when the fluctuations first start becoming unimportant below $T_{c}$ (the Ginzburg criterion [15]) and where the mean field theory approach starts becoming applicable. Fluctuations in $\phi$ have an insignificant effect on the thermodynamics of the phase transition if [15]:

$$
\tau>>\tilde{T}_{c}^{2}
$$

where $\tilde{T}_{c} \equiv m T_{c} / \eta^{2}$. We refer to the temperature when the above condition is near saturation as the Ginzburg temperature. This temperature, which is essentially defined when the change in free energy $\Delta F$ between the two vacua in a correlation volume is comparable to the temperature, serves as a benchmark for the temperature $T_{\text {st }}$ at which structure becomes well-defined as the fluctuation rate between vacua is $\Gamma_{\text {fluct }} \propto e^{-\Delta F / T}$, and below the Ginzburg temperature the rate is exponentially supressed. A naive comparison of the expansion rate $\Gamma_{\text {exp }}$ of the Universe with the fluctuation rate, where the exponential prefactor is constructed using dimensional analysis, indicates that structure at, or not too far below, $T_{G}$ is essentially "frozen in" (there is still tension in the walls, however, which will smooth out structure below the freeze-out temperature $T_{s t}$, see, e.g., Ref. [16] ). In what follows, we shall assume that $T_{s t} \leq T_{G}$, and only explore equilibrium properties below the Ginzburg temperature; we mention, however, that it might be possible for the undetermined dimensionless prefactor to alter our conclusion.

To be more precise about the Ginzburg temperature, which by its very nature is somewhat indeterminate, we shall define $T_{G}$ by a condition equivalent to $\Delta F \sim T$, yet expressed in terms of statistical properties of $\phi$, which are directly obtainable from our simulations: 


$$
\left\langle(\phi-\langle\phi\rangle)^{2}\right\rangle /\langle\phi\rangle^{2} \simeq 1
$$

The above definition is very appropriate, as the criterion (2.12) can be derived by comparing the width of the probability distribution $p\left(\phi_{\text {corr }}\right)$, where $\phi_{\text {corr }}$ are values of $\phi$ obtained in a correlation volume, with the average value of $\phi$. Since our lattice spacing is in fact a correlation length, Fig. 3 can be used to determine the Ginzburg temperature: $\tilde{T}_{G} \simeq 0.0994$. We performed three runs on a $30 \times 30 \times 30$ lattice at this temperature. [From Fig. 4 we note that at $T_{G} \simeq 0.0994$ the total wall area is comparable to the maximum allowed value on the lattice, i.e., the width of the walls are comparable to their separation.] The distribution of the $P_{i, j, k}$ 's after a run is shown in Fig. 5. It can be seen that the width of the distribution is indeed comparable to the average value of $P$. The total wall area, averaged over three runs, associated with each wall size is shown in Fig. 6. It is clear from the figure that most of the wall area is associated with the smaller walls. An unusual feature of this graph are tiny peaks, first appearing for a wall area of $16 \xi^{2}$, surrounded by much larger ones. This can be explained by the fact that configurations with surface area $\neq\left(2+4 V / \xi^{3}\right) \xi^{2}$, where $V$ is the volume enclosed by the domain wall, first start appearing when the wall area is $16 \xi^{2}$ (the configuration corresponding to a minimization of surface area of 4 connected cubes).

The first simulation of domain walls, in a cosmological context, was performed by Harvey et al. [17]. They randomly assigned + and - signs to the cells of a lattice, and examined the resulting structure. An identical non-dynamical simulation of the formation of domain walls was also performed by Vachaspati and Vilenkin [5]. When comparing results, we shall compare with Ref. [5], as they made the interpretation that the lattice spacing should roughly be a correlation length (based upon work done by Kibble [2]), leading to the presently accepted scenarios of wall and string formation. It is well known from percolation theory [18] that if lattice sites are "marked" by an $X$ with probability $p_{X}$, then a cluster of $X^{\prime} s$ will percolate through the lattice for $p_{X}$ above a critical probability $p_{c}$. For a cubic lattice $p_{c} \simeq 0.31$, which means that both + and - clusters will percolate in such a simulation, and that large-scale domain walls will always exist. The results from such a simulation are indisputable if there are practically no correlations in the sign of $\phi$ on scales $\approx \xi$. However, it is clear from our previous work that in thermal equilibrium, at least for temperatures $T \leq T_{G}$, either + or - signs, but not both, percolate through the lattice and an interpretation of the lattice spacing in Ref. [5] as a correlation length at the Ginzburg temperature is inappropriate.

The lack of both + and - percolation at the Ginzburg temperature can be seen from mean-field theory. In the Gaussian approximation we have: $d p / d P=\exp (-[P-$ 
$\left.\bar{P}]^{2} / 2 W^{2}\right) / \sqrt{2 \pi} W$, where $W^{2}=\left\langle(P-\bar{P})^{2}\right\rangle$, and $\bar{P}=\langle P\rangle$. Choosing the vacuum state with $\bar{P}>0$, the probability of finding a + site is then:

$$
p_{+}=[1+\operatorname{erf}(\bar{P} / \sqrt{2} W)] / 2
$$

where $\operatorname{erf}(x)$ is the error function. Applying condition (2.13), we find $p_{+}=[1+$ $\operatorname{erf}(1 / \sqrt{2})] / 2 \simeq 0.84$, and hence $p_{-} \simeq 0.16$. This indicates that the - sites will not percolate through the lattice at (or below) the Ginzburg temperature. It is important to note that a transition from small-scale domain structure to large-scale domain structure occurs at a temperature $T_{L S}$ not too much larger than our definition of the Ginzburg temperature (2.13). We estimate where this transition occurs by setting $p_{+}=1-p_{c} \simeq 0.69$ in Eqn. (2.14), finding that $\bar{P} / W \simeq 0.5$. The relationship between $\bar{P} / W$ and temperature, or $\Delta F / T$, is easily estimated by neglecting the spatial gradient term in the free energy and fitting a Gaussian, centered at $\bar{P}$, to $e^{-V_{T} V_{\xi} / T}$, where $V_{\xi}$ is a correlation volume. The result is that $\bar{P}^{2} / W^{2}=\lambda \eta^{4} \tau^{2} V_{\xi} / 3 T=8 \Delta F / T$, where $\Delta F$ is the change in free energy between the $P=\bar{P}$ and $P=0$ states. [To consider $\Delta F$ between the two distinct vacua, the spatial gradient term cannot be ignored. Estimating the gradient term in the free energy as $(\phi-\langle\phi\rangle)^{2} V_{\xi} / 2 \xi^{2}$, we find $\bar{P}^{2} / W^{2} \simeq 3 \Delta F / 2 T$.] Since $\Delta F / T \approx \bar{P}^{2} / W^{2}$, we see that at $T_{L S}$ the exponential supression in the fluctuation rate has really not "kicked in" yet, and our definition of the Ginzburg temperature is an appropriate, conservative one. [Recall that it is the freeze-out temperature which is really relevant, and which we expect to be below our definition of the Ginzburg temperature.] It is clear that $T_{L S}$, expressed in terms of parameters in the potential, must have the same scaling as the Ginzburg temperature. This fact has also been recently noticed based upon other considerations [19].

It should not be a surprise that a simulation of the type of Vachaspati and Vilenkin cannot describe the equilibrium distribution of domain walls, even though the only length scale that arises in the simulation is $\xi$. In general, the correlation length is not a measure of a length over which correlation volumes are uncorrelated with respect to the sign of $\phi$-it is just a measure of the typical scale of spatial fluctuations of the field. In addition, if one simply assigns +'s and -'s at random, with equal probability, the $+\leftrightarrow-$ symmetry is manifest, indicating that such a simulation can only make sense if $T \geq T_{c}$, or if the fields are not completely thermalized (e.g., if there are horizons). The present situation indicates a fundamental problem with the Monte Carlo simulationit is too simplistic. In equilibrium, at the Ginzburg temperature, all the structure is essentially small-scale. However, we also know that large-scale structure must result due to causality being limited to horizon scales. If the correlation length and horizon scales are disparate, as was originally intended, it seems very hard to rationalize their scenario. 


\section{B. Cooled Fields}

We now attempt to unify both (equilibrium) small-scale and (non-equilibrium) largescale structure by dynamically simulating the phase transition, on time scales that allow horizons in our box. Structure can then be examined at $T_{G}$. There are a number of ways one could do this, but we choose the simplest possible procedure: (1) as initial conditions we take $P_{i, j, k}, R_{i, j, k}=0,(2)$ we set $T=T_{G}$, and (3) we input the desired horizon size to correlation length ratio $r$, from which the number of time steps of the simulation are calculated. So, the initial conditions represent a state of complete $\phi \leftrightarrow-\phi$ symmetry $(\psi=0)$. By setting $T=T_{G}$, and running the simulation on time scales $t_{r u n}$ smaller than the box size $L$, local regions of broken symmetry will develop on length scales $\sim t_{r u n}$, but the symmetry will be globally restored as the vacuum state in each local region is randomly determined by thermal fluctuations.

We first provide an estimate of $r$ at the Ginzburg temperature. The critical temperature of the phase transition is estimated to be $T_{c} \simeq 2 \eta$ [9]. The correlation length is $\xi_{G} \simeq m^{-1} / \sqrt{\tau_{G}} \sim m^{-1} / \tilde{T}_{c} \sim m^{-1} / \sqrt{\lambda}$, where $\tau_{G}$ is obtained from Eqn. (2.12). Assuming the transition occured during the radiation dominated era, the horizon size at $T_{G}$ is $d_{G}=0.6 g_{*}^{-1 / 2} m_{p l} / T_{G}^{2}$, where $m_{p l}=1.22 \times 10^{19} \mathrm{GeV}$ (the Planck mass), and $g_{*}$ is the effective particle degrees of freedom $\left(g_{*}=106.75\right.$ in the standard model). Assuming perturbative self-interactions $(\lambda<O(1))$ we have $T_{G} \simeq T_{c}$, and hence $r=d_{G} / \xi_{G} \simeq 0.1 \lambda g_{*}^{-1 / 2} m_{p l} / T_{c}$. For a GUT scale transition $T_{c} \sim 10^{15} \mathrm{GeV}$ and we see that $r<O(100)$ for $\lambda<O(1)$, i.e., the horizon is probably not significantly larger than the correlation length for theories of greatest interest. However, we note for theories with transition temperatures $T_{c}<<T_{G U T}, r$ can be a very large number. Explorations of such theories can be found in Ref. [20], where strings form at the electroweak scale $\left(T_{c} \sim 10^{2} \mathrm{GeV}\right)$ and $r \simeq 10^{16} \lambda$.

If $r$ is close to unity, the Monte-Carlo type simulation of Vachaspati and Vilenkin should accurately describe the domain wall (and string) distribution at $T_{G}$. [Note that freeze-out is determined here by a comparison of the fluctuation rate with the damping rate, which has been taken as a constant $\Gamma=3 m_{\text {eff }}$ throughout. Again the Ginzburg temperature is relevant for providing a benchmark temperature at which freeze-out occurs.] Therefore, we consider the case $r>1$. Unfortunately, this requires that we use a lattice with three disparate length scales $\xi_{G}, d_{G}$, and $L$ which significantly reduces the range of parameter space that can feasibly be studied. We therefore only consider the case $r=10$. As domain walls are cosmologically disastrous over a wide range of parameters [1], we only qualitatively describe the results of the simulation and reserve a more detailed analysis for global strings, in the next section. We performed a number of simulations on both $40^{3}$ and $50^{3}$ lattices, and found in 
each case that both + and - clusters percolated through the entire lattice resulting in one 'gigantic' domain wall which represented about $75 \%$ of the total wall energy. In simulations with + and - signs randomly distributed on the lattice sites, about $90 \%$ of the total wall area is associated with one large-scale domain wall. That we obtain a smaller percentage of the total area in the infinite wall (by 'infinite', we mean a wall whose spatial extent is comparable to the box size) is not too surprising as our equilibrium results indicated a strong preference towards smaller walls.

\section{Formation of Global Strings}

\section{A. Equilibrated Fields}

We now consider the formation of global $U(1)$ strings (for a good review of cosmic strings, see [16]). These strings are described by a complex scalar field $\sigma$ and a zerotemperature potential with a degenerate set of vacuum states connected by global phase transformations $\sigma \rightarrow e^{i \nu} \sigma$, which we take to be $V(\sigma)=\lambda\left(|\sigma|^{2}-\eta^{2} / 2\right)^{2} / 3$ !. It is known that such a field theory admits cylindrically symmetric string solutions $[21,22]$ where the phase $\chi$ of the Higgs field $\sigma\left(=f e^{i \chi} / \sqrt{2}\right)$ winds $N$ times in the space of Higgs vacua as the fields are examined in configuration space through a rotation of $2 \pi$ in the angular coordinate $\theta$. The string solutions with $N=-1,1$ are topologically stable, as the $N=0$ state cannot be reached by a continuous transformation of the Higgs fields; global strings with $|N| \geq 2$ are unstable to decay into $|N|$ vortices of unit vorticity [23]. The classical field theory solution for the radial profile $f(r)$ of a string of unit vorticity cannot be found analytically, but has the properties $f(0)=0$, $f(\infty)=\eta$ and a simple variational calculation [23] yields a string width $w \approx 1.3 m^{-1}$, where $m$ is the Higgs mass. Again we shall consider a temperature-dependent effective potential of the form: $V(\Phi)=-m^{2} \tau|\Phi|^{2}+\lambda|\Phi|^{4} / 3 !+$ constant $\left[\tau \equiv 1-\left(T / T_{c}\right)^{2}\right]$, where $\Phi$ is the classical part of $\sigma$. The probability of a given configuration $\{\Phi\}$ is given by $p \propto e^{-F / T}$, where $F$ is the usual Ginzburg-Landau free energy functional. The most probable field configuration is then given by $|\Phi|^{2}=\eta^{2} \tau / 2$ for $T<T_{c}$, and $|\Phi|^{2}=0$ for $T>T_{c}$.

The formulae of the last section are easily generalized if we use the representation $\Phi=\left(\Phi_{1}+i \Phi_{2}\right) / \sqrt{2}$, where $\Phi_{1}$ and $\Phi_{2}$ are real fields. The zero-temperature Higgs

mass has the same form as before, i.e. $m=\sqrt{\lambda} \eta / \sqrt{2}$, and we can define the same dimensionless parameters as in Sec. I with the following modifications: $P_{s} \equiv \Phi_{s} / \eta$, $R_{s} \equiv \partial P_{s} / \partial \tilde{t}$ where $s=1,2$. The difference equations $(2.7),(2.8)$ are then generalized 
to:

$$
\begin{aligned}
P_{i, j, k}(\tilde{t}+\epsilon)_{s}= & P_{i, j, k}(\tilde{t})_{s}+\epsilon R_{i, j, k}(\tilde{t})_{s} \\
R_{i, j, k}(\tilde{t}+\epsilon)_{s}= & R_{i, j, k}(\tilde{t})_{s}-\epsilon\left(G_{i, j, k}(\tilde{t})_{s}+\tilde{\Gamma} R_{i, j, k}(\tilde{t})_{s}\right. \\
& \left.+P_{i, j, k}(\tilde{t})_{s}\left[P_{i, j, k}^{2}(\tilde{t})_{1}+P_{i, j, k}^{2}(\tilde{t})_{2}-\tau\right]\right)+\alpha_{i, j, k}(\tilde{t})_{s}
\end{aligned}
$$

where $G_{i, j, k}(\tilde{t})$, and $\alpha_{i, j, k}(\tilde{t})$, have the same form as in Sec. I, but with an index $s$, where $s=1,2$.

As before, we start by mapping out phase structure. Here we define the order parameter $\psi$ as:

$$
\psi=|\langle\Phi /(\eta / \sqrt{2})\rangle|=\sqrt{\left\langle P_{1}\right\rangle^{2}+\left\langle P_{2}\right\rangle^{2}}
$$

and again $\psi=1$ characterizes a state of maximum disorder, and $\psi=0$ describes a state with the global $\Phi \rightarrow e^{i \nu} \Phi$ symmetry intact. We use the same lattice spacing as before, i.e. $\tilde{\Delta}=1 / \sqrt{\tau}$, and again use a $20 \times 20 \times 20$ lattice to explore phase structure. The results are shown in Figs. 7 and 8.

The number of strings of a given length was also calculated at various temperatures. We defined a string through a given lattice face if the phase of the Higgs field changed by $\pm 2 \pi$ in a closed path around the face. The total string length on the $20^{3}$ lattice is shown as a function of temperature in Fig. 9. Averages, and uncertainties, were calculated from three statistically independent field configurations. The total string length rapidly increases near the critical temperature, and the transition to the ordered $\psi=0$ state can be viewed as proceeding through the increased production of strings. Inside each string the symmetry is restored, and when the separation of strings becomes indistingushable from their widths the symmetry is restored globally.

The Ginzburg criterion for global strings is the same form as in Eq. (2.12), but again we shall remove the indefiniteness associated with the $>$ sign, and define $T_{G}$ by the condition:

$$
\left\langle(\Phi-\langle\Phi\rangle)^{*}(\Phi-\langle\Phi\rangle)\right\rangle /\left\langle\Phi^{*}\right\rangle\langle\Phi\rangle=\left[\left\langle P_{1}^{2}\right\rangle+\left\langle P_{2}^{2}\right\rangle-\psi^{2}\right] / \psi^{2} \simeq 1
$$

which is equivalent to setting the change in free energy of a uniform vacuum state $|\Phi|^{2}=\eta^{2} \tau / 2$ to a state with a $\Phi=0$ fluctuation of size $\simeq \xi_{G}^{3}$ comparable to the temperature. From Fig. 8 we see that $T_{G} \simeq 0.0987$. From Fig. 9 it can be seen that the probability of a string passing through a randomly chosen lattice face at this temperature is $\simeq 0.05$. This probability is about a factor of 6 smaller than that obtained from randomly assigning Higgs phases to the lattice sites. We performed 
three runs on a $30^{3}$ lattice at this temperature. The total loop length, averaged over three runs, associated with each loop size is shown in Fig. 10. The total string length at $T_{G}$ is in very small loops, and no infinite strings were found. Because of the low loop density (relative to the Monte Carlo simulations) and limited lattice size, it was not considered worthwhile to fit data and find other statistical quantities as in Ref. [5]. For example, it would not be useful to try obtaining a fractal dimension of the strings when the largest loop in the simulation has only 28 segments.

To conclude our discussion of the equilibrium properties of global strings, we compare our results with related works. Numerical studies of the equilibrium properties of strings $[24,25]$ have been performed by solving the Nambu equations of motion for a 'gas of strings' in a box. Here the total energy is a fixed parameter of the simulation. At low energy densities (small compared to the only scale for the energy density $\mu^{2}$, where $\mu$ is the energy per length of the string) it was found that an initial configuration of strings will chop itself up into a large number of small loops with a typical size of order the lattice cutoff. Infinite strings were not found below a critical density $\rho_{c} \sim \mu^{2}$. Above $\rho_{c}$, long strings appeared and as $\rho$ is increased $>\rho_{c}$ most of the energy density is found in long strings. These results have also been obtained analytically [26] by quantizing the string and counting states in the microcanonical ensemble. Similar results were found here, quantified in terms of the temperature. A transition from small-scale strings to large-scale strings occurred not far above the Ginzburg temperature. In analogy with the domain wall case, where we analytically argued that a phase transition from small-scale to large-scale structure takes place at a temperature $T_{L S} \propto T_{G}\left(T_{G}<T_{L S}<T_{c}\right)$, we expect the same behaviour.

Copeland et. al. [19] have also analytically studied the behaviour of loops and long strings, in the Abelian-Higgs model, through the phase transition. They found, in thermal equilibrium, that most of the energy was in long strings at a temperature $T_{*}$, which they defined to coincide with structure formation. They also showed that $T_{*}$ was of order the Ginzburg temperature. However, $T_{*}$ was defined as the temperature above which the loop and infinite-string partition function diverged. At temperatures just below $T_{*}$, loops quickly (exponentially) dominated the percentage of the total energy. We disagree with their interpretation of $T_{*}$ as the temperature at which strings formed, but their results relate the same physics as that found here.

Finally, we should remark that we have considered quite different degrees of freedom than those studied in the aforementioned works. Also, we have examined global strings, which have long-range interactions, whereas local, Nielsen-Olesen [21] strings were studied in Ref. [19], and Nambu strings were studied in Refs. [24,25,26]. We note, however, that our results for global strings might be more general than they 
appear. At the Ginzburg temperature the width of global strings are comparable to their seperation, which effectively limits the range of interaction at formation to a correlation length, i.e. the global strings behave like local strings.

\section{B. Cooled Fields}

Now, we follow the same procedure as in the previous section to examine both small-

scale and large-scale structure. Here, $P_{i, j, k}(\tilde{t}=0)_{1}=P_{i, j, k}(\tilde{t}=0)_{2}=0$ were chosen as initial conditions so that the phase of the Higgs field was initially undefined, everywhere. Again, we only consider the case $r=10$. We performed a number of runs on $40^{3}$ and $50^{3}$ lattices. The results of two such runs are shown in Figs. 11 and 12. Most of the string length ( $>50 \%$ ) is found to occur in the smaller loops, rather than in large, infinite strings. This is in contrast to the results from the Monte-Carlo simulations, where it was found that $80 \%$ of the total string length was in infinite strings. Again, we can view our results in terms of the equilibrium configuration of strings shifting the distribution of energy to the smaller scales.

A few words about the meaning of these results are in order. We have not examined the dependence of the results upon our definition of the freeze-out temperature, which should be determined numerically. Also, a more appropriate choice of initial conditions would be a thermal distribution, which could be obtained using the procedure of the last subsection. Further improvements are listed in the conclusion. Such improvements are not warranted within the framework presented here, as a proper analysis of the full-blown problem should be done in an expanding spacetime. The important point is that we have determined that there is no current, realistic, model describing the formation of walls or strings when the thermal correlation length at freeze-out is significantly smaller than the horizon.

\section{Concluding Remarks}

Using a simple formalism, we have been able to study both equilibrium and nonequilibrium properties of global strings and domain walls. We numerically verified the Kibble mechanism for the production of topological defects, and shed new light on the details of the formation process. We found that in equilibrium, for temperatures $T \leq T_{G}$, most of the energy was associated with defects of the smallest size, and there was no infinite structure. For a horizon size ten times larger than the correlation length, we found that at the Ginzburg temperature, in both the domain wall and 
global string case, energy was shifted (relative to the Monte-carlo simulations) from the large scales to the small scales. We have presented results for only one choice of $\tilde{T}_{c}$, but other values were studied and the same qualitative behaviour was observed. The presently held view that $80 \%$ of the string length at formation is in long strings deserves the qualification that it is only true if the correlation length at the Ginzburg temperature is comparable to the horizon length. Otherwise, such a simulation would be highly questionable due to the assumption of random phases on correlation length scales. The degree of randomness is crucial in determining statistical properties. The equilibrium phase transition from large-scale to small-scale structure near the Ginzburg temperature illustrates just how sensitive the string distribution can be to an assumption about the phase distribution.

It is interesting to note that the same arguments used to describe the formation of strings in the 'standard scenario' were actually also used to show that monopole production could be highly supressed in the early Universe [27]. We have found that neither of these conflicting scenarios can be completely correct. In Ref. [27] they showed monopoles could be suppressed due to thermal fluctuations, without taking into account horizons. Their results are similar to those we obtained for walls and strings when we examined equilibrium properties at the Ginzburg temperaturewe found no 'infinite' walls or strings. However, one cannot claim that monopoles, strings, or walls can be cosmologically insignificant without a proper treatment of horizons.

We mention that a major question remains unanswered. What happens to the string distribution at formation in the limit that the horizon size to correlation length ratio becomes very large $(r \rightarrow \infty)$, as in low temperature phase transitions $\left(T_{c}<<\right.$ $\left.m_{p l}\right)$ ? This limit is certainly not equivalent to the case where just the equilibium configuration applies (no infinite strings); rather, it is the limit where there are finitesize horizons with $\xi / d \rightarrow 0$. If we assumed a power law in the distance scales for the ratio of the energy in small loops to that in long strings, an extrapolation of our results would indicate that small loops would quickly dominate the energy distribution of strings in this limit. A naive way to consider this limit might be to perform a nondynamical Monte Carlo type simulation involving two scales, rather than one. For example, in the domain wall case, one might randomly assign + and - vacua to cubic cells representing horizon volumes. Then a simulation on a lattice with spacing equal to the correlation length, within each horizon cell, could be performed by laying down + and - signs with probabilities $p_{+}$and $p_{-}$depending upon the vacuum state of a given horizon and the desired temperature. One might be led to believe that the total area in the infinite walls at $T_{G}$ is then a surface phenomenon while the total area associated with the finite walls is a volume effect. 
Finally, we call attention to a number of improvements that could be made on the work presented here: (1) the expansion of the Universe can easily be taken into account and, in this regard, the potential can also be varied according to the temperature-time relationship of the Universe; (2) the details of the noise term can be improved, and physically motivated; (3) it would be useful to numerically find out when freeze-out occurs, rather than using the Ginzburg temperature as a benchmark; (4) a systematic study of statistical properties, such as the fractal dimension of infinite strings at the Ginzburg temperature, would be of interest; (5) a careful study of the string distribution with different ratios of horizon size to correlation length at formation may lead to an acceptable extrapolation of the formation details for the case $\xi / d<<1 ;(6)$ lastly, it would be interesting to perform a similar analysis for local strings. A number of these items shall be incorporated into a future work [28].

Note Added. The two-scale Monte Carlo model mentioned above was recently examined for arbitrary freeze-out temperatures, and several values of $r$ [29]. It is found that the contribution of large-scale walls to the total wall density $\sim r^{-1}$ near the Ginzburg temperature, in qualitative agreement with the results found here. This seems to be a geometrical effect, in which case one might argue that large-scale strings contribute a fraction $\sim r^{-2}$ of the total string density near the Ginzburg temperature. Such geometrical arguments might explain why large-scale structure decreased relatively less for walls than strings in simulations with the the same value of $r$.

\section{Acknowledgements}

We thank Andy Albrecht and Edmund Copeland for useful discussions. Special thanks go to Rocky Kolb for his careful reading of the manuscript, and useful criticism. Finally, we thank Michael Turner for support and encouragement. This work was supported in part by the DoE (at Chicago) and for computing by the NASA and DoE (at Fermilab). This work is submitted in partial fulfillment of the requirements for a Ph.D. in physics at the University of Chicago. We acknowledge NASA grant number NAGW-1340 at Fermilab. 


\section{References}

[1] Ya. B. Zel'dovich, I. Yu Kobzarev and L.B. Okun, Zh. Eksp. Teor. Fiz. 67, 3 (1974) [Sov. Phys. JETP 40, 1 (1975)].

[2] T.W.B. Kibble, J. Phys. A 9, 1387 (1976); Phys. Rep. 67, 183 (1980).

[3] Ya. B. Zel'dovich, Mon. Not. Roy. Astron. Soc. 192, 663 (1980); A. Vilenkin, Phys. Rev. Lett. 46, 1169, 1496 (E) (1981); R.H. Brandenberger, A. Albrecht and N. Turok, Nucl. Phys. B277, 605 (1986).

[4] A. Albrecht and N. Turok, Phys. Rev. Lett. 54, 1868 (1985); D.P. Bennett and F.R. Bouchet, Phys. Rev. Lett. 60, 257 (1988).

[5] T. Vachaspati and A. Vilenkin, Phys. Rev. D30, 2036 (1984).

[6] A. Albrecht and N. Turok, Phys. Rev. Lett. 54, 1868 (1985).

[7] R. Scherrer and J. Frieman, Phys. Rev. D33, 3556 (1986).

[8] N. Turok, Fermilab-CONF-88/116-A (1988).

[9] D. Kirshnitz, JETP Lett. 15, 529 (1972); D. Kirshnitz and A. Linde, Phys. Lett. B 42, 471 (1972); L. Dolan and R. Jackiw, Phys. Rev. D 9, 3320 (1974); S. Weinberg, Phys. Rev. D 9, 3357 (1974).

[10] H. Risken, The Fokker Planck Equation [Springer Verlag] (1984).

[11] R. Loft and T. DeGrand, Phys. Rev. B35, 8528 (1987).

[12] G.G. Batrouni, G.R. Katz, A.S. Kronfeld, G.P. Lepage, B. Svetitsky and K.G. Wilson, Phys. Rev. D32, 2736 (1985).

[13] F. Graziani and K. Olynyk, Fermilab Report 85/175-T, 1985 (unpublished); R. Brandenberger, H. Feldman and J. MacGibbon, Phys. Rev. D37, 2071 (1988); H. Feldman, Phys. Rev. D38, 459 (1988).

[14] S. Ma, Modern Theory of Critical Phenomena [Benjamin] (1976).

[15] V.L. Ginzburg, Sov. Phys. Solid State 2, 1824 (1960).

[16] A. Vilenkin, Phys. Rep. 121, 263 (1985).

[17] J.A. Harvey, E.W. Kolb, D.B. Reiss and S. Wolfram, Nucl. Phys. B201, 16 (1982). 
[18] D. Stauffer, Phys. Rep. 54, 1 (1979).

[19] E. Copeland, D. Haws and R. Rivers, to appear in Nucl. Phys. B.

[20] A. Vilenkin, Phys. Rev. Lett. 53, 1016 (1984); E. Chudnovsky and A. Vilenkin, Tufts preprint TUTP-88-1.

[21] H. Nielsen and P. Olesen, Nucl. Phys. B61, 45 (1973).

[22] A. Vilenkin and A.E. Everett, Phys. Rev. Lett. 58, 1867 (1982).

[23] C.T. Hill, H.M. Hodges and M.S. Turner, Phys. Rev. D 37, 263 (1988).

[24] A.G. Smith and A. Vilenkin, Phys. Rev. D 36, 990 (1987).

[25] M. Sakellariadou and A. Vilenkin, Phys. Rev. D 37, 885 (1988).

[26] D. Mitchell and N. Turok, Phys. Rev. Lett. 58, 1577 (1987); Nucl. Phys. B294, 1138 (1987).

[27] F. Bais and S. Rudaz, Nucl. Phys. B170, 507 (1980).

[28] H. Hodges, in preparation (1989).

[29] H. Hodges, U.C. Santa Cruz preprint SCIPP 89/11. 


\section{Figure Captions}

1. The total string length in loops of a given size, as a function of loop size, given by the non-dynamical Monte Carlo model. This simulation was done on a $35^{3}$ cubic lattice with periodic boundary conditions. There are four 'long' strings in this particular simulation, which represent $\simeq 80 \%$ of the total string length.

2. The order parameter $\psi$ as a function of the dimensionless temperature $\tilde{T}$. Above $\tilde{T}_{c} \simeq 0.1$ the $\phi \leftrightarrow-\phi$ symmetry is restored. The solid line is the mean-field result $\bar{P}=\sqrt{\tau}\left(T>T_{c}\right)$.

3. The fluctuations $\left\langle(P-<P>)^{2}\right\rangle /\langle P\rangle^{2}$ as a function of the dimensionless temperature $\tilde{T}$.

4. The total number of domain wall segments on a $20^{3}$ lattice as a function of temperature $\tilde{T}$.

5. The distribution of fields $P_{i, j, k}$ in thermal equilibrium after a run on a $30^{3}$ lattice at the Ginzburg temperature.

6. The total wall area in walls of a given size, at the Ginzburg temperature, as a function of wall size.

7. The order parameter $\psi$ as a function of the dimensionless temperature $\tilde{T}$. Above $\tilde{T}_{c} \simeq 0.1$ the global $\Phi \rightarrow e^{i \nu} \Phi$ symmetry is restored. The solid line is the meanfield result $\bar{P}=\sqrt{\tau}\left(T>T_{c}\right)$.

8. The fluctuations $<(\Phi-<\Phi>)^{*}(\Phi-<\Phi>)>/\left\langle\Phi^{*}>\langle\Phi\rangle\right.$ as a function of the dimensionless temperature $\tilde{T}$.

9. The total string length on the $20^{3}$ lattice as a function of the dimensionless temperature $\tilde{T}$.

10. The total string length in loops of a given length, at the Ginzburg temperature, as a function of string length.

11. The total string length in loops of a given length on a $40^{3}$ lattice, at the Ginzburg temperature with horizons at ten times the lattice spacing, as a function of string length.

12. The total string length in loops of a given length on a $50^{3}$ lattice, at the Ginzburg temperature with horizons at ten times the lattice spacing, as a function of string length. 


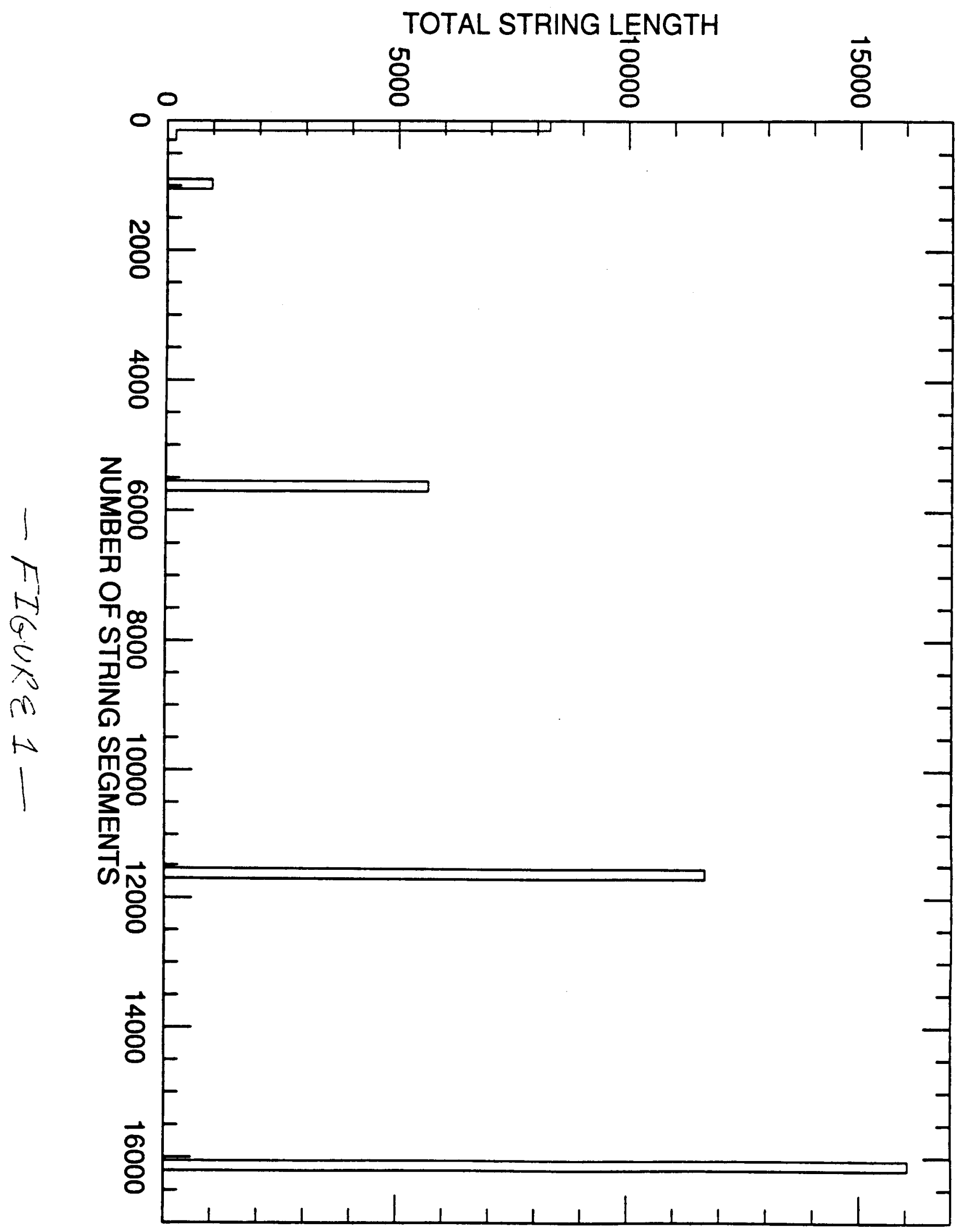




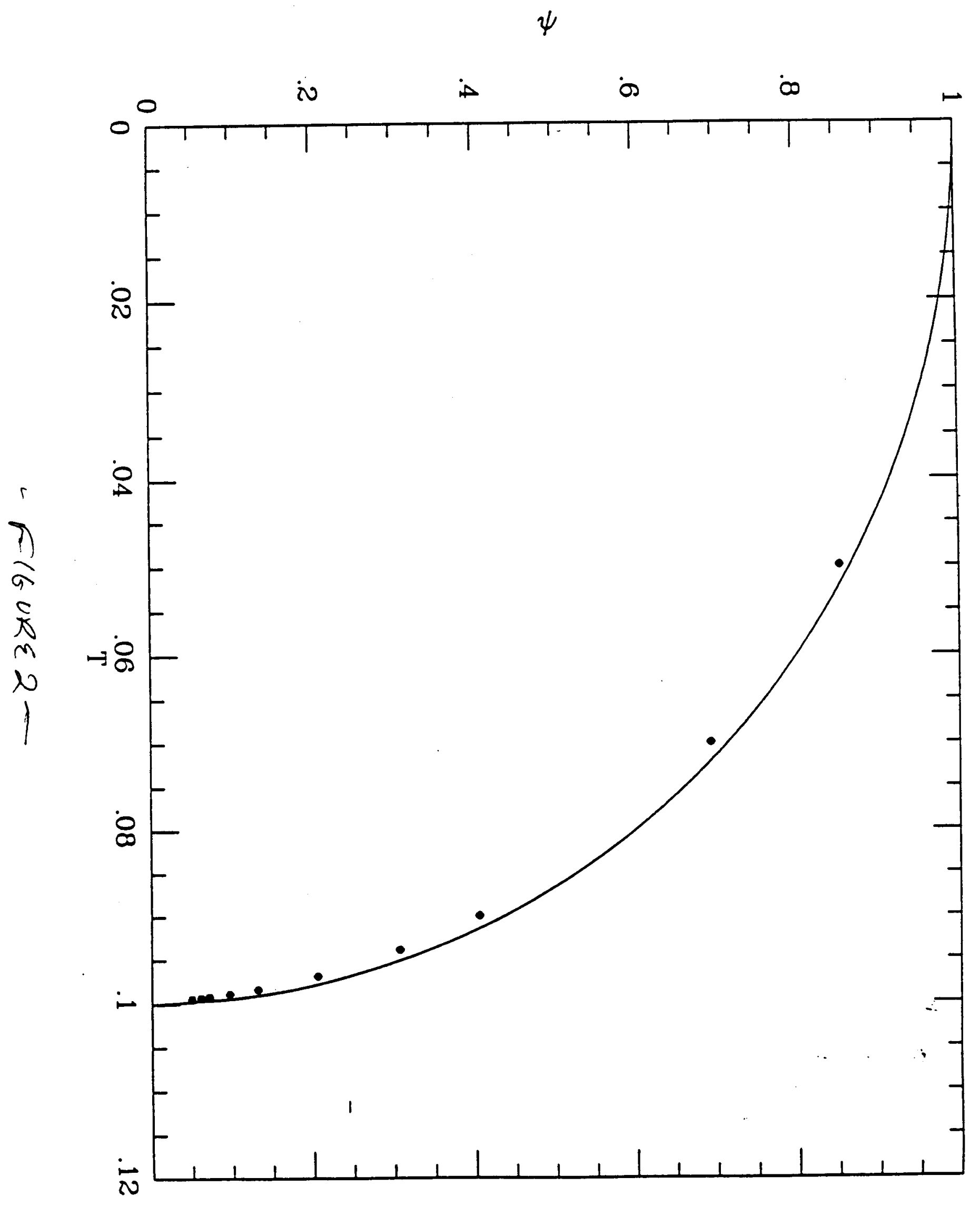


Fluctuations

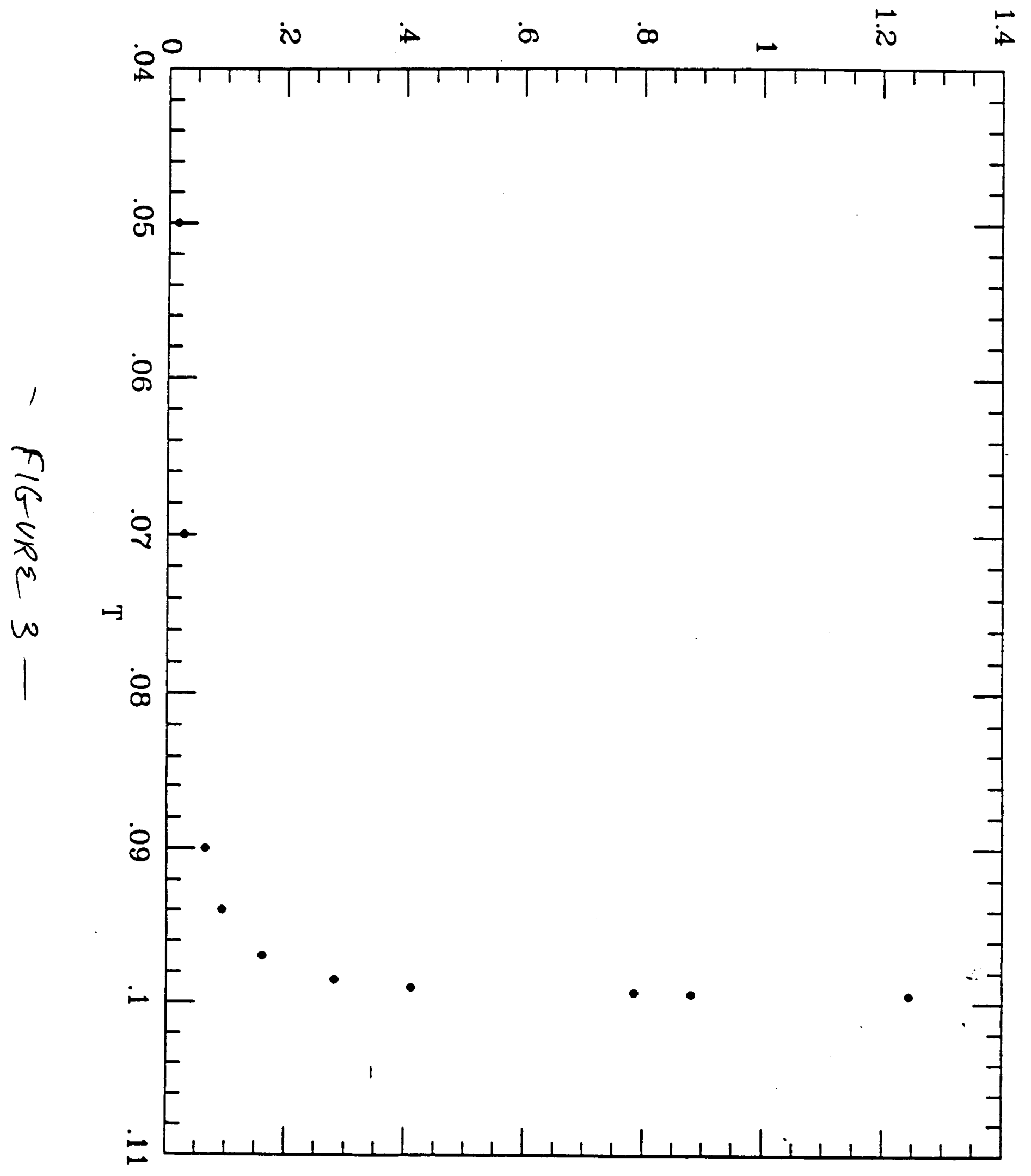




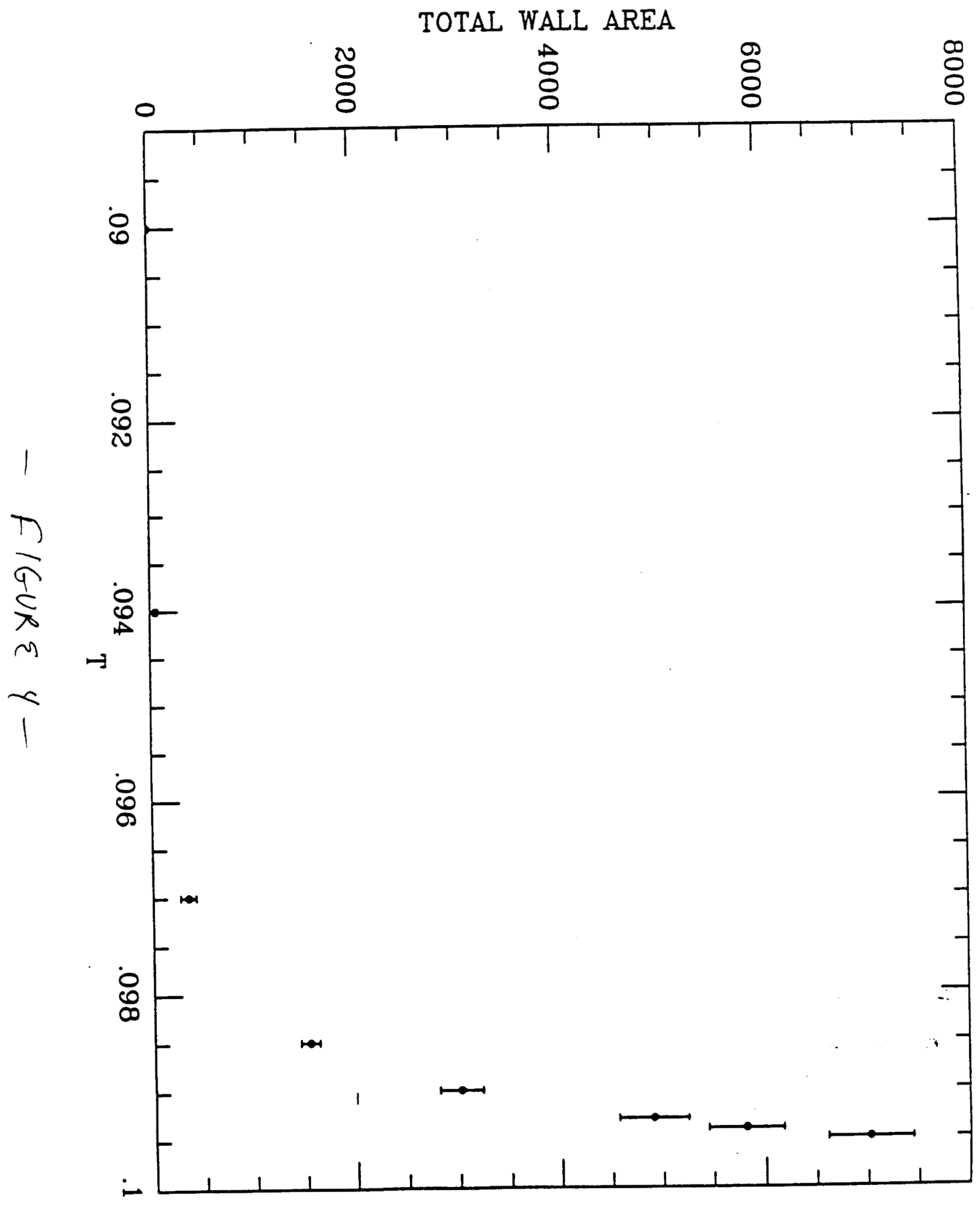




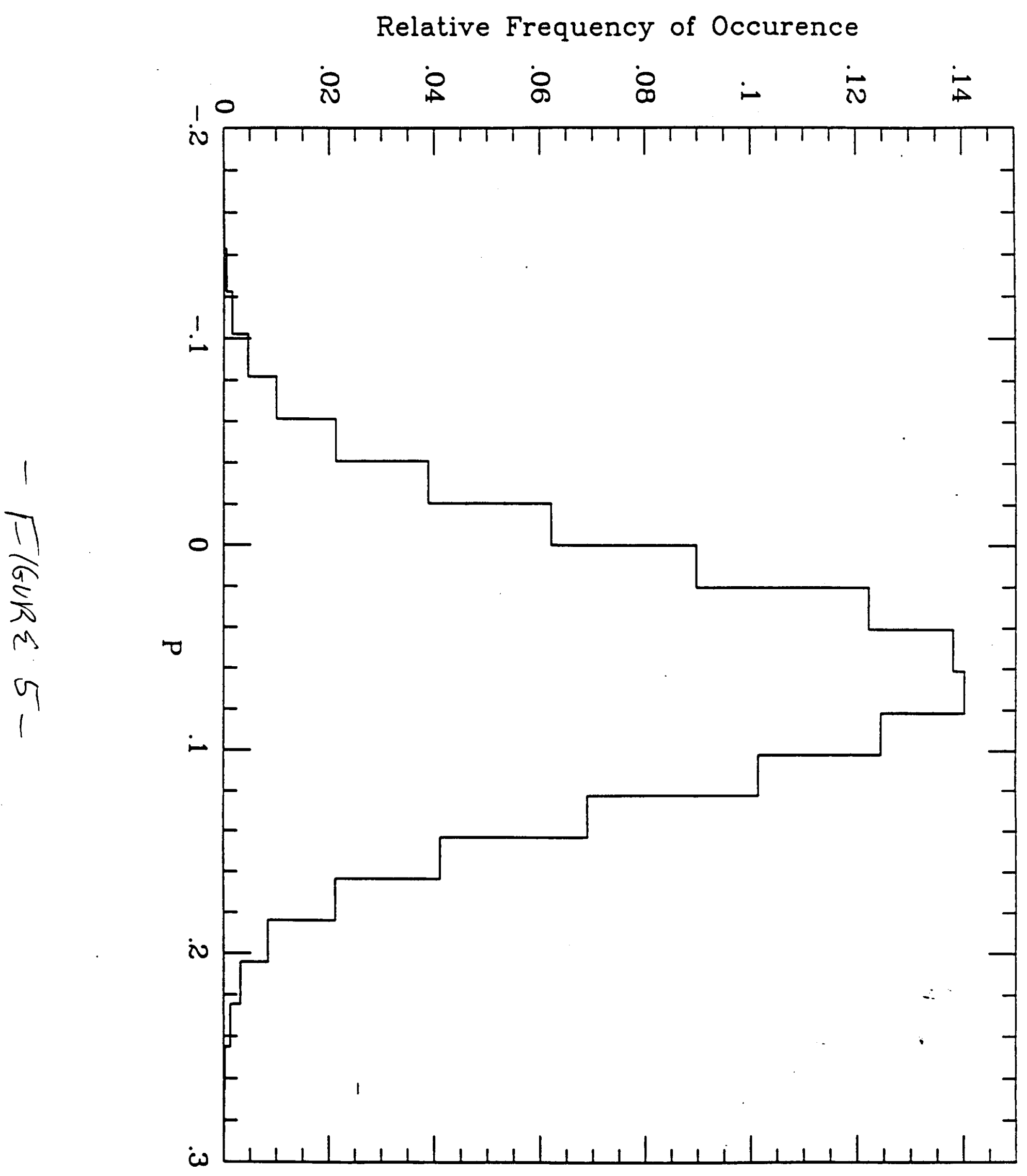


TOTAL WALL AREA

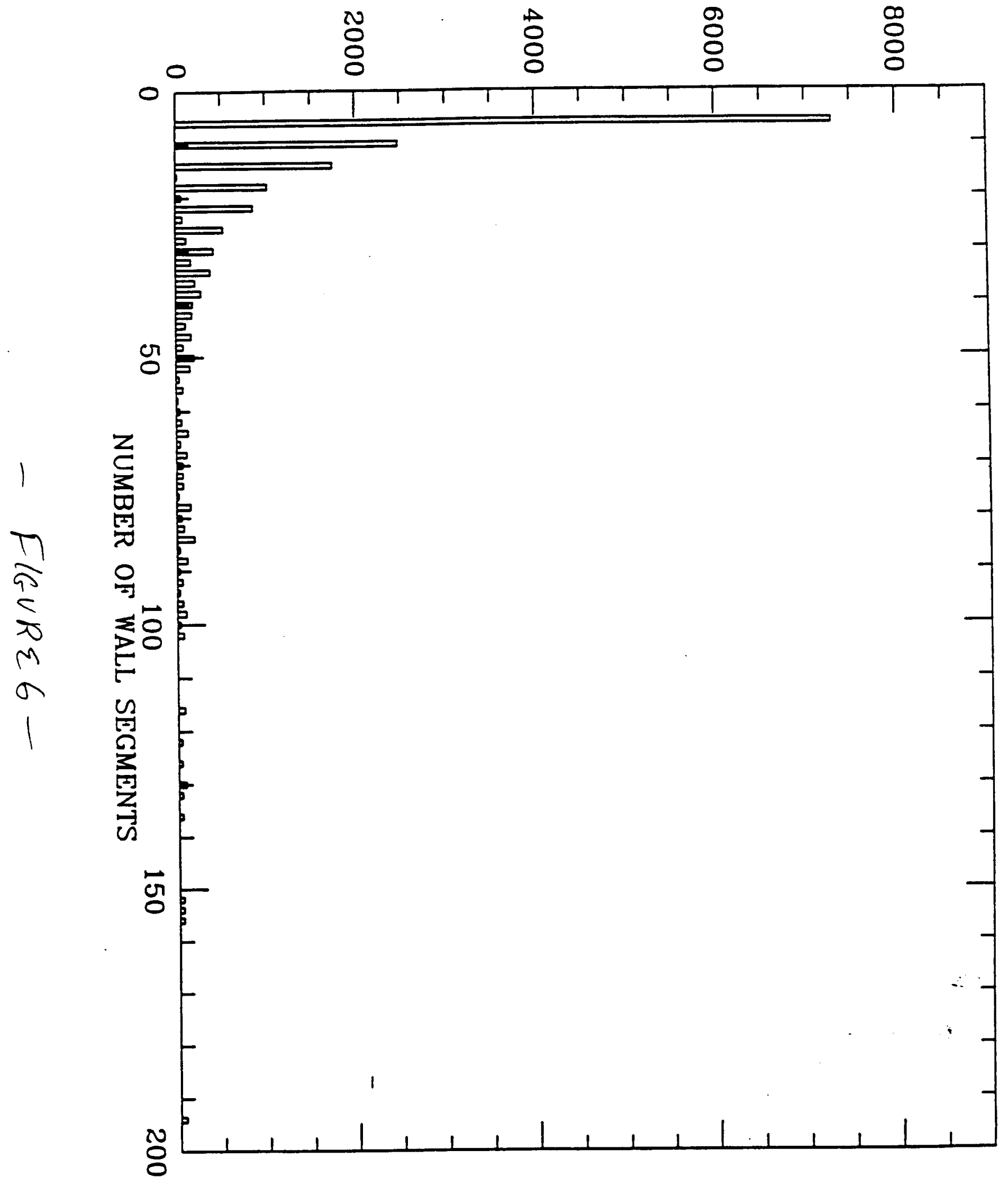




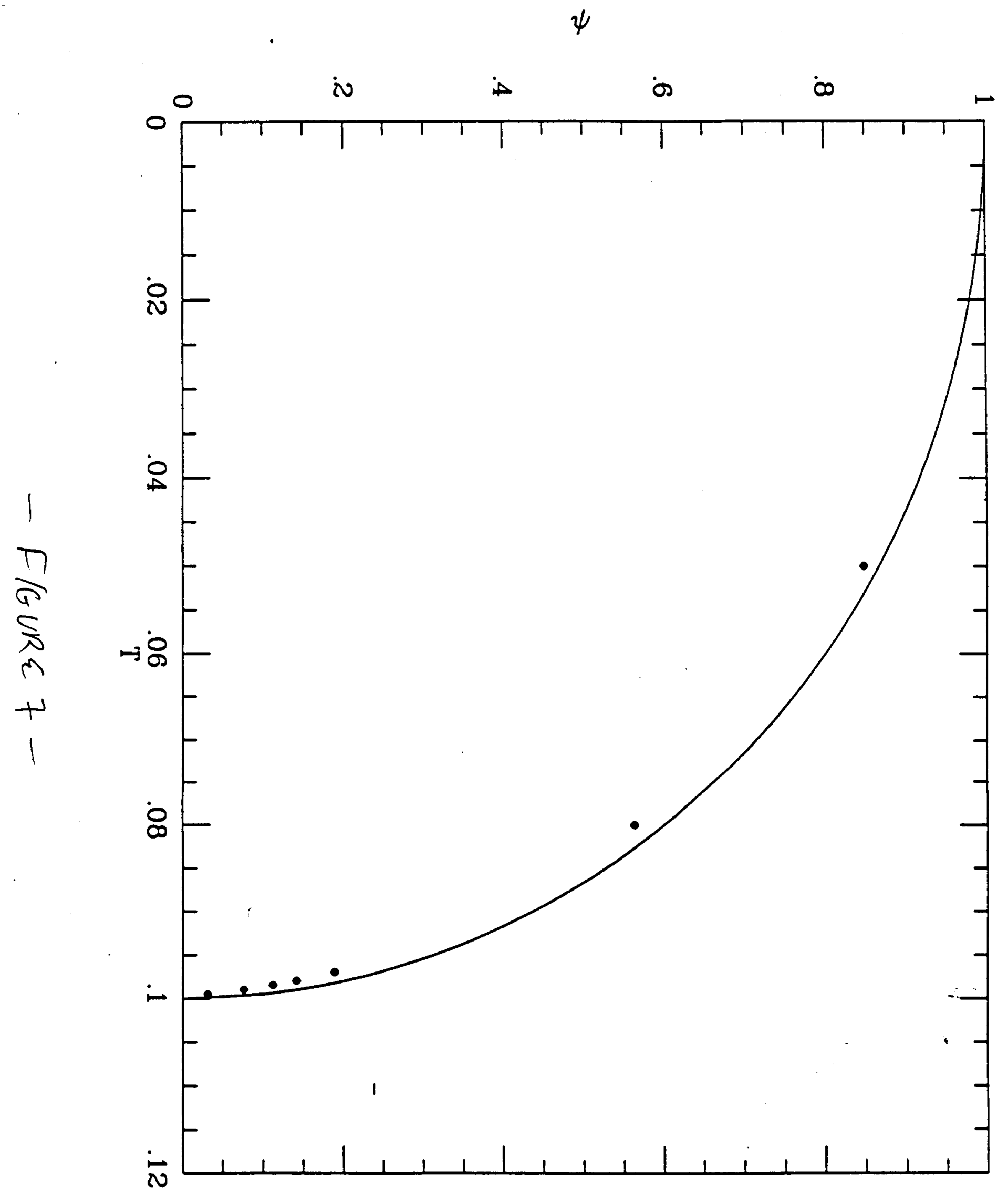


Fluctuations

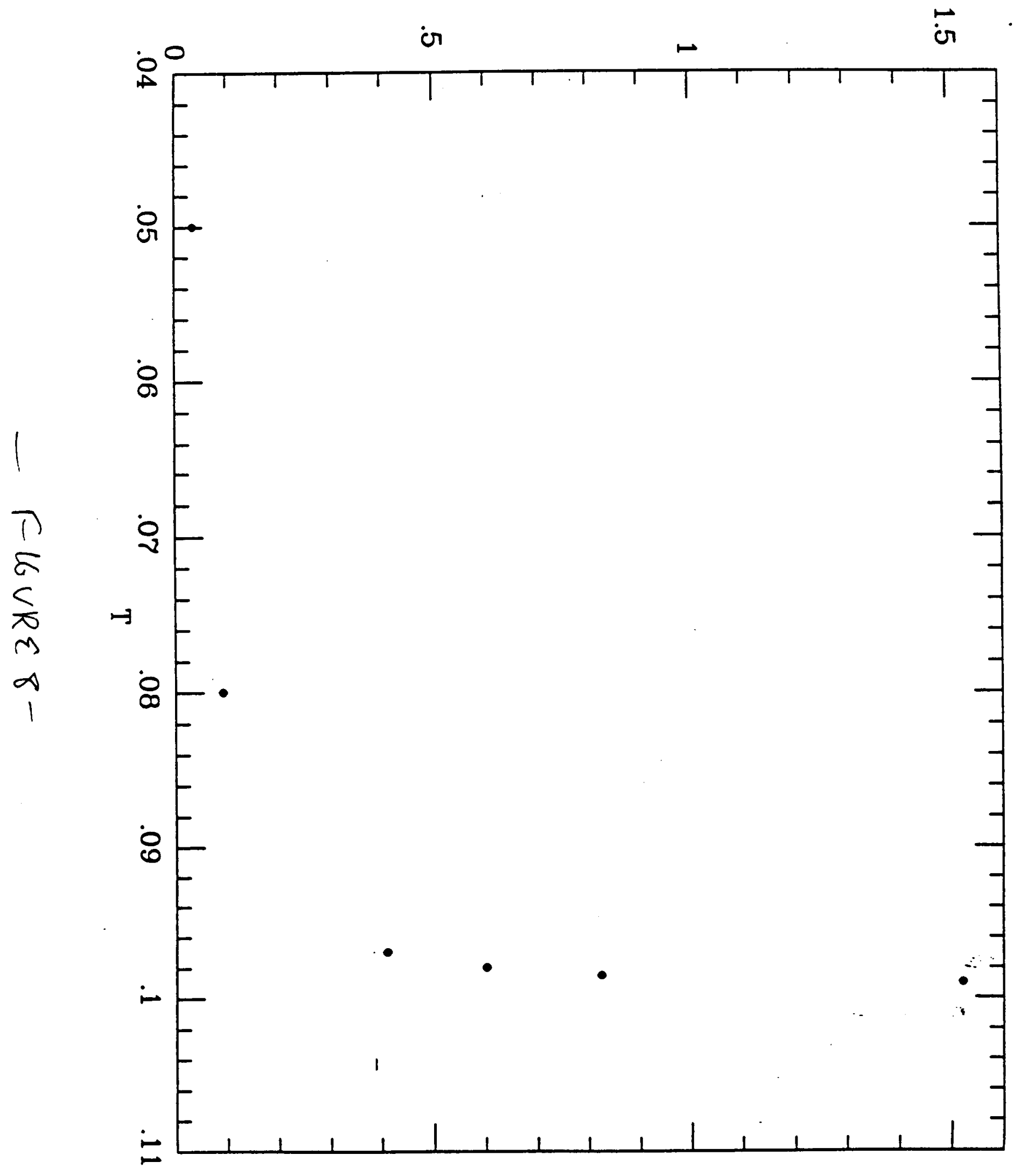

